\title{
Theoretical model of structure-dependent conductance crossover in disordered carbon
}

\author{
Mikhail V. Katkov and Somnath Bhattacharyyd** \\ Nano-Scale Transport Physics Laboratory and School of Physics, \\ University of the Witwatersrand, Private Bag 3, WITS 2050, Johannesburg, South Africa
}

\begin{abstract}
We analyze the effects of $s p^{2} / s p^{3}$ bond-aspect ratio on the transport properties of amorphous carbon quasi- $1 D$ structures where structural disorder varies in a very non-linear manner with the effective bandgap. Using a tight-binding approach the calculated electron transmission showed a high probability over a wide region around the Fermi-level for $s p^{2}$-rich carbon and also distinct peaks close to the band edges for $s p^{3}$-rich carbon structures. This model shows a sharp rise of the structure resistance with the increase of $s p^{3} C \%$ followed by saturation in the wide bandgap regime for carbon superlattice-like structures and suggests the tuneable characteristic time of carbon-based devices.
\end{abstract}

Introduction: Amorphous carbon $(a-C)$ thin films have long been considered as very important materials for understanding the fundamentals of electronic structure [1-6] and mechanical properties [7-9]. Depending on the preparation method, a very diverse nature of microstructure can be formed ranging from diamond-like to graphite-like carbon. Significant efforts have been made to explain the optical properties of $a-C$ films based on the estimated band gap and degree of disorder 10 18]. There are also numerous interesting results showing a structure-dependent conductivity crossover in $a-C$ films 19 22]. However, a rigorous theoretical model of electrical transport of $a-C$ films related to the carbon microstructure is yet to be developed that can explain the experimentally observed highly non-linear variation of the conductivity with $s p^{2}$ bond concentration [12, 18]. In carbon the electronic properties can be strongly influenced by bond disorder which depends on the $s p^{2} / s p^{3}$ bond aspect ratio and on the $s p^{2}$ cluster size. Although there is no well defined structure for $a-C$ films they have been described in many studies as a mixture of $s p^{2}$ and $s p^{3}$ hybridized bonds [10-15, 18]. A number of spectroscopic techniques, for example Raman, optical absorption and photoelectron spectroscopy, have confirmed this assertion [2, 8, 14, 15]. It was found that the size of the $s p^{2}-C$ clusters greatly influenced the bandgap [2]. The optical absorption edge was described by a Gaussian plot based on the normal distribution of cluster size [23]. Films with low $s p^{2}$ concentrations have also been described as quasi- $1 D$ polymer chains (e.g. transpolyacetylene) [3, 15-17, 23, 24]. The effect of $1 D$ filamentary channels on electronic transport has been observed in low-dimensional $a-C$ films and related devices [25]. However, no significant theoretical studies have yet been undertaken to investigate the tunneling properties unlike in $1 D$ molecular structures [26]. Based on previous studies we believe that $a-C$ films can be described effectively as a distribution of periodical alternation of the hopping energy and constant energy term. Starting from previous experimental claims for the variation of disorder

\footnotetext{
* Corresponding author somnath.bhattacharyya@wits.ac.za
}

with the energy gap [12, 14, 15], we establish the trend of localization length in a wide range of carbon films based on the calculated transmission coefficients $T(E)$ and local density of states (LDOS). In this Letter we propose a Gaussian disorder analogous to previous studies [12, 14, 15] but in a different manner which is directly connected to the microstructure of $a-C$ films. Since the nature of topological disorder has not been clearly understood from previous works we briefly discuss this effect on electron transmission at the end of this Letter.

Proposed Structure: We develop a quasi $1 D$ dimensional $a-C$ superstructure, which can be represented by a network of narrow nano-ribbons with a length of $87 \AA$ for the zigzag and $75 \AA$ for the armchair directions (Fig. 1(a)d. This structure is a quasione-dimensional representation of disordered $a-C$, for similar examples see [3, 27, 28] which include some of the essential features specifically $s p^{2} / s p^{3}$ clusters and bond angle distortions. There are 4 segments of the $s p^{2}$ structure with widths ranging from 1 to 10 sites depending on the phase percentage. The superstructure is a mix of saturated and conjugated $\left(s p^{3}-s p^{2}\right)$ areas, corresponding to $\sigma$ and $\pi$ bonds. The percentage of $s p^{2}$ carbon structures determining the average $s p^{2}$ cluster size [2] can be promoted, for example by nitrogen doping. The effects of the lateral dimensions can be taken into account by considering quantization of the wave vector in this direction and modifying the hopping terms in the longitudinal direction.

Methodology: We use a tight-binding Hamiltonian describing electrons confined in $q-1 D a-C$ ribbons as

$$
H=\sum_{n} \epsilon_{n} c_{n}^{\dagger} c_{n}-\sum_{n} t_{n+1, n}\left(c_{n+1}^{\dagger} c_{n}+c_{n}^{\dagger} c_{n+1}\right),
$$

where $\epsilon_{n}$ is an on-site energy, which can represent an atomic energy as well as an external potential. $c_{n}^{\dagger}$ and $c_{n}$ are operators of creation and annihilation of electrons. $t_{n+1, n}$ is a hopping term between site $n$ and $n+1$, which takes into account nearest-neighbor hopping that can be different from site to site reflecting the structural change. We seek the solution of the Schrödinger equation

$$
E \psi_{n}=\epsilon_{n} \psi_{n}-t_{n+1, n} \psi_{n+1}-t_{n-1, n} \psi_{n-1}
$$


on the tight-binding lattice with the known eigenvalue $E=\epsilon_{l}-2 t \cos k_{l}$, where the unit cell length is fixed to 1. $t_{l}$ and $\epsilon_{l}$ are the hopping term and on-site energy, respectively, corresponding to the left lead, which can be interpreted as the Fermi energy $\left(E_{f}\right)$, and $k_{l}$ is an incoming wavevector. The boundary conditions imply that there is only an outgoing plane wave at the right lead, while at the other sites in the tight-binging lattice there are incident and reflected plane waves. Starting from a unique outgoing wave on the right lead $\psi_{n}=e^{i k_{r} n}$, we backpropagate this solution to obtain $\psi_{1}$ and $\psi_{0}$ using Eq. (2). We get the incoming wave amplitude $A$ and define $T(E)=\frac{1}{|A|^{2}} \frac{\sin k_{l}}{\sin k_{r}}$, (outgoing wavevector, $k_{r}$ ). We calculate the transmission coefficient as a function of incident electron energy, where the transmission coefficient is the ratio of the outgoing to incoming probability current (since $k$ vectors may be different for incoming and outgoing waves). Thus, the problem is different from that of finding the eigenvalues as the transmission coefficient is evaluated as a function of energy. The resonance energies determined from transmission maxima correspond to eigen-energies of the system.

The Carbon model: In this model a Hamiltonian with alternating values of the resonance integral produces conduction and valence bands as well as a band gap [26]. For the saturated region we introduce the resonance integral alternation by the hopping terms $t_{\min }^{\sigma}$ and $t_{\max }^{\sigma}$. In the presence of the conjugated compound the effective gap decreases because of the electron resonance at energies lying inside the $s p^{3}$ phase band gap. The hopping term $t_{\pi}$ corresponding to the $s p^{2}$ phase experiences distortion due to the structural disorder, which reflects the difference in the bond length via the deformation potential. Topological disorder corresponding to the difference in the cluster size affects the resonance conditions via the boundary conditions for quasi-bound states [15]. The disorder of the $s p^{3}$ phase is neglected due to its minimal effect on the resonant electron transmission within the gap [2]. The broadening effects are mostly due to disorder in the well. As a matter of fact the resonant states have a larger amplitude inside the wells in this range of energies. The leads on the right and left sides are taken as ordered narrow $1 D$ graphite-type strips. The incoming electron energies are given with respect to the $\pi$ electron $\left(s p^{2}-C\right)$ on-site energy $\epsilon_{\pi}=0 \mathrm{eV}$. Therefore, the position of the valence and conduction $s p^{3}$ phase is not symmetric due to the different on-site energies for $\pi$ and $\sigma$ electrons and has a shift corresponding to the difference in these energies, as shown in Fig. 1(b). The hopping between $s p^{3}$ and $s p^{2}$ hybridized $C$-atoms is extremely significant due to the non-planar geometry of the $s p^{3}$ structure 7]. We use the following tight-binding parameters: $s p^{3}$ on-site energy $\epsilon_{\sigma}=-0.9 \mathrm{eV}, s p^{2}-s p^{2}$ transfer integral: $t_{2-2}=3.40 \mathrm{eV}$, $s p^{2}-s p^{3}$ transfer integral $t_{\sigma-\pi}=1.37 \mathrm{eV}, s p^{3}-s p^{3}$ transfer integrals $t_{\sigma}^{\min }=1.10 \mathrm{eV}$ and $t_{\sigma}^{\max }=4.30 \mathrm{eV}$ [26, 29]. Fig. 1(b) shows the levels of hopping terms along with on-site energies for atoms in $s p^{3}(\sigma)$ and $s p^{2}$ $(\pi)$ structures.

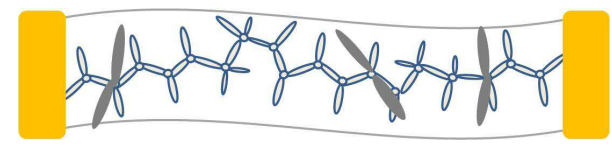

(a)

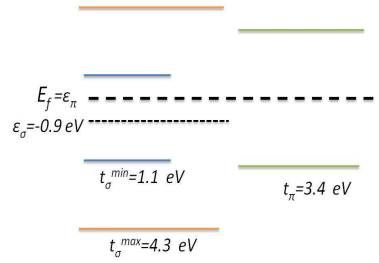

(b)

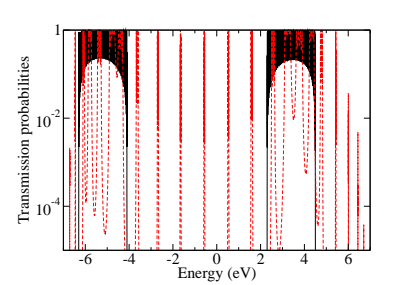

(c)
FIG. 1. (a) Microstructure of $a-C$ consists of $\sigma$ and $\pi$ bonded carbon atoms. (b) The band diagram shows $s p^{3}$ hopping term alternation (red and blue lines online) and $s p^{2}$ hopping term level. The longer dashed line corresponds to $s p^{2}$ on-site energy, which is taken as the zero level, the shorter dashed line corresponds to $s p^{3}$ on-site energy. (c) $T(E)$ vs. incident electron energy $(E)$ showed a number of quasi-bound states of mainly conjugated $\left(78 \% s p^{2}\right)$ (dashed curve, red on-line) compared to pure saturated $\left(s p^{3}\right)$ structure (solid curve).

Fig. 1(c) shows $T(E)$ of a pure saturated structure and an ordered structure with $78 \%$ of $s p^{2}$ bonding concentration where the zero level corresponds to $s p^{2}$ on-site energy. The numerically calculated band gap and band widths of the saturated structure are $E_{\sigma}^{g}=6.4 \mathrm{eV}$ and $\Delta E_{\sigma}^{c(v)}=2.2 \mathrm{eV}$, respectively. For the mixed structure, there are several narrow peaks within the $s p^{3}$ band gap corresponding to quasi-bound states of the $s p^{2}$ "wells". The peaks are very sharp with a high peak-to-valley ratio. In the case of a symmetric structure, $T(E)$ always reaches 1 [26]. When the concentration of $s p^{2}$ structure is high, the peaks are close to the $E_{f}$. On the other side of the concentration limit (low $s p^{2}$ phase), the peaks are close to the band edges of $s p^{3}$ structure. Fig. 2(a) shows the first resonance peak position $E_{\text {res }}$ (from the zero energy level) as a function of $s p^{2}$ phase percentage, which has an inverse square dependence on the percentage. Such a dependence reflects a linear increase of the average $s p^{2}$ cluster size and a quadratic decrease of the energy associated with the resonance.

Effect of Disorder: There is a significant effect of structural $s p^{2}$ disorder on the transport properties of the $s p^{3}-s p^{2}$ carbon system. Disorder is an inherent micro-structural property of $a-C$ films. It has been suggested that the density of the bonding and anti-bonding $\pi$ states could be represented with a Gaussian distribution 8, 15, 23]. In particular the Urbach energy was described as a function of the width of the $\pi$ DOS peak at $E_{f}$ although the link between disorder and the broadening parameter was not clear. The disorder parameter $\delta$, which takes account of bond length (angle) distortions, was calculated previously [15]. Micro-structural disorder is broadly separated into structural and topological disorder although the later only applies for a large $s p^{2}$ clus- 
ters. In this Letter a typical hopping disorder parameter $\delta$ for a Gaussian distribution of the hopping term $t_{\pi}$ is shown in Fig. 2(b) for 3 cases, A, B and C as a function of $E_{\pi}$, which covers the major possible behaviors of disorder in carbon structures. Models B and C are suggestions for the behavior of the disorder parameter based on the assumption that the disorder parameter can either increase or decrease with the $E_{\pi}$ corresponding to either $s p^{3}$ rich or $s p^{2}$ rich structures. The values of the disorder parameter for model A $\delta$ can be extracted from a number of experiments (e.g., Raman $G$-peak linewidth) [12, 14, 15] and we believe it is the result of two competing processes (B and $\mathrm{C}$ ). On this basis, we have determined the behavior of the disorder parameter as a function of $E_{\pi}$. We therefore focus on model $\mathrm{A}$ as it corresponds to the physical nature of $a-C$ systems. A number of $s p^{2}-C$ percentages were chosen to cover a wide range. The corresponding $E_{\pi}$ values (and hence corresponding values for $\delta$ ) were determined based on the relationship between $s p^{2}-C \%$ and $E_{\pi}$. We found that the values of $E_{\pi}$ were very close to the values of $E_{\text {res }}$. Here $2 E_{\pi}$ is the peak-to-peak energy spacing of bonding and antibonding DOS above and below the $E_{f}[15]$. For case A, the disorder reaches its maximum at about $E_{\pi}=1.9 \mathrm{eV}$, followed by a slight decrease. This is an attribute of the structural relaxation, which occurs (also for case B) when $s p^{2}$ clusters become relatively large [2, 3]. For case $\mathrm{C}$ the disorder increases sharply with $E_{\pi}$ corresponding to the
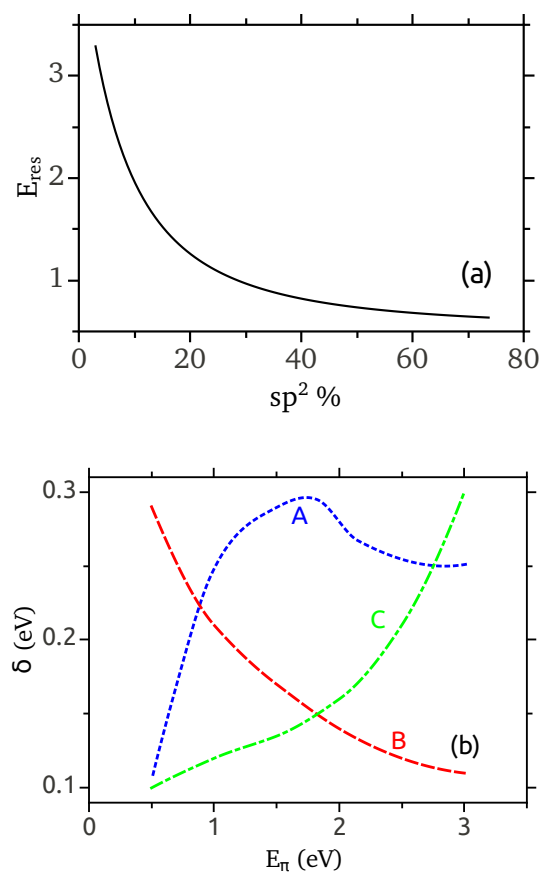

FIG. 2. (a) First resonance peak from the zero level $E_{\text {res }}$ as a function of $s p^{2}$ phase percentage. (b) Hopping disorder parameter $\delta$ of the $s p^{2}$ region for cases $\mathrm{A}, \mathrm{B}$ and $\mathrm{C}$ as a function of $E_{\pi}$, where $2 E_{\pi}$ is the peak-to-peak energy spacing of bonding and antibonding states. rise of $s p^{3}$ phase content. In our further calculations we associate $E_{\pi}$ with the energy of the first (from zero) resonance peak, $E_{\text {res }}$. The proposed non-linear graph (case A) showed a significantly different trend of $T(E)$ and localization length compared with the two other cases where a continuous decrease (B) or increase (C) of $\delta$ with $E_{\pi}$ is considered.

In Fig. (3) (a) we show $T(E)$ for $73 \% s p^{2}$ bond concentration, which corresponds to $2 E_{\text {res }}=1.1 \mathrm{eV}$ and Gaussian disorder $\delta=0.10 \mathrm{eV}$, averaging the values over a set of 500 runs. Other cases are shown in Fig. 3 (b) $42 \%$, $2 E_{\text {res }}=1.7 \mathrm{eV}, \delta=0.19 \mathrm{eV}$, (c) $24 \%, 2 E_{\text {res }}=2.2 \mathrm{eV}$, $\delta=0.26 \mathrm{eV}$ and (d) $7.8 \%, 2 E_{\text {res }}=3.7 \mathrm{eV}, \delta=0.29 \mathrm{eV}$. With the decrease of $s p^{2}$ bond concentration and increase of disorder in model $\mathrm{A}$, we observe an increase in the $2 E_{\text {res }}$ value (the effective band gap), broadening of the peak width and a decrease in peak-to-valley ratio. With further decrease of $s p^{2}$ bond concentration, the resonance peaks move closer to the $s p^{3}$ band gap and finally disappear. Models $\mathrm{A}$ and $\mathrm{C}$ show significant broadening of the resonant peaks compared to case B for low $s p^{2} \%$ since disorder is large (see Fig. 3(c) and (d), insets) although it is very similar for all cases for high $s p^{2} \%$. At large $s p^{2}$ percentages the transmission through the structure is high therefore structural effects only slightly influence the transmission coefficient. When the $s p^{2}$ percentage is low, the transmission is also low hence even slight structural changes have a large effect on $T(E)$.

The disorder induces localization of the wave function within the structure and the localization length is expressed as $L_{l o c}\left(E_{r e s}\right)=-\frac{2 L}{\ln T\left(E_{r e s}\right)}$, where $L$ is the length between leads used in the Landauer formula for calculating the resistance 24]. At small energies $L_{l o c}$ is longer for case A and C than for case B because the structure is more transparent at resonant energies for lower disorder (Fig廿4(a), lower curves). The localization length decreases sharply for all 3 cases till $E_{\text {res }}$ reaches the value of about $1 \mathrm{eV}$ with a further saturation showing an exponential behavior. The resistance behaves oppositely to the localization length (Fig廿(a), upper curves). At small energies the resistance is smallest for case $\mathrm{C}$ and largest for case $\mathrm{B}$ following the distortion parameter dependence. The structural resistance increases till $E_{\text {res }}=2 \mathrm{eV}$ followed by a slight decrease at higher energies in the natural logarithm scale. This value is related to the mobility edge- as the disorder parameter increases, the transmission maxima shift away from $E_{f}$ towards the mobility edge. The lifetime of resonant states therefore decreases (the full width at half maximum (FWHM) decreases). At large energies it is greater for case A and C (almost coincide) because of the larger distortion values. From this analysis it is revealed that the tunnel conductance can be improved in $s p^{2}$-rich carbon for model $\mathrm{C}$ and also in $s p^{3}$-rich carbon for model B. Whereas for models A the conductance can vary as intermediately between model $\mathrm{B}$ and $\mathrm{C}$. Although the tunnel resistance increases initially in the low energy region it can be controlled for a wide band gap carbon structure. In practice the size of 

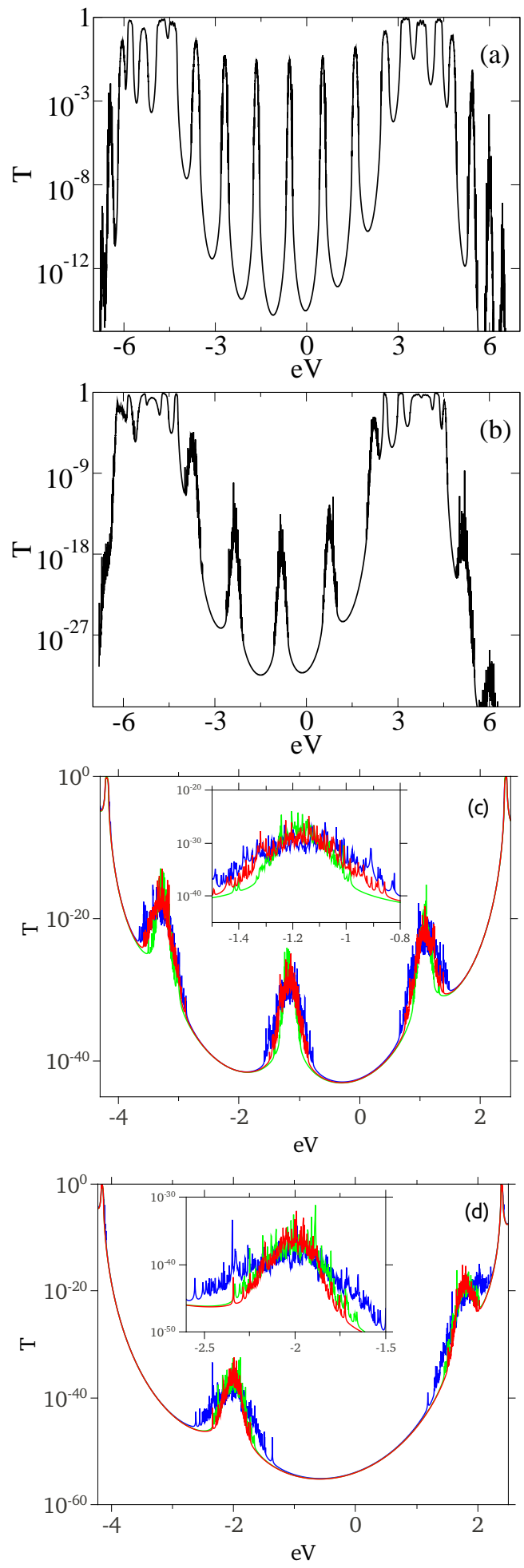

FIG. 3. $T(E)$ vs. $E$ for the following $s p^{2}$ bond concentration: (a) $72 \%$,(b) $42 \%$,(c) $24 \%$, and (d) $7.8 \%$. In subfigures (c) and (d) 3 curves (in color online) correspond to 3 cases of hopping disorder parameter $\delta$ dependance on $E_{\pi}$ as shown in Fig. 2(b). The tight-binding parameters are the same as that in Fig. 1(c) The peaks for Model A showed a broader feature than that of case B and C, shown in the insets of Fig. (c) and (d).
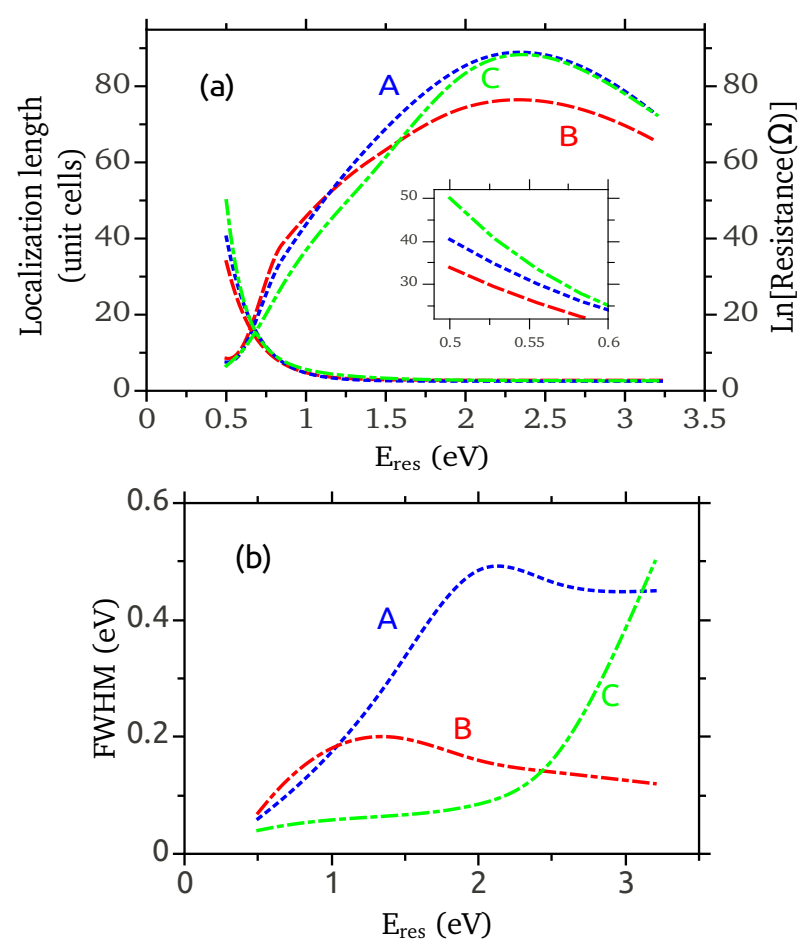

FIG. 4. (a) Localization length (lower curves) and resistance (upper curves) calculated at $E_{\text {res }}$ peaks, which are determined by the factor of disorder as well as the energy distance from the $s p^{3}$ structure band edge. The inset shows $L_{l o c}$ at low energies. (b) Variation of FWHM for the transmission peaks $E_{\text {res }} .3$ curves (in color online) correspond to cases A, B and $\mathrm{C}$ of hopping disorder parameter $\delta$ dependance on $E_{\pi}$ as shown in Fig[2(b).

the $s p^{2}$ clusters can be controlled to some extent through processes such as annealing or irradiation hence the band gap can be controlled to some extent. Nitrogen doping (discussed below) is also a possibility. These results explain the initial increase of resistance of tetrahedral $a-C$ $(t a-C)$ films incorporated with a small amount of nitrogen followed by a decrease for high nitrogen $\left(\right.$ or $\left.s p^{2}-C\right)$ concentration [18].

A comparative study of the FWHM for cases A, B and $\mathrm{C}$ showed a significant difference not only in their absolute values but also in the their trends as a function of energy. For case A, the FWHM increases from low energy followed by a saturation at energies above $2.5 \mathrm{eV}$. For case B, the FWHM decreases beyond $1.5 \mathrm{eV}$ (due to decrease of disorder). For case C, the FWHM increases continuously above $2.5 \mathrm{eV}$. The other factors which effect the FWHM are the incoming energy $E_{\text {res }}$ and the width of the $s p^{3}$ "barrier". A higher energy and smaller width increase the peak width due to the higher transparency of the structure for an incoming wave. The value of the FWHM, characterizing an average quasi-bound state lifetime at a particular energy, approaches saturation in the high $s p^{3}$ limit. The width of the resonant peaks is propor- 
tional to the characteristic time of resonant states therefore as the disorder increases, the FWHM of resonant peaks and consequently the characteristic time of resonant states also increases. At the same time the amplitude of transmission maxima decreases rapidly. The conductance crossover occurs as with further decrease of the $s p^{2}$ bond concentration the resonant peaks shift towards the mobility edge and the characteristic time therefore decreases with the resistance decreasing accordingly. The FWHM of the resonant peaks saturate at very low $s p^{2}$ bond concentration but the resistance does not saturate as the amplitude of the resonance peaks increases as the peaks approach the mobility edge. These results clearly establish the specific effect of the non-linear model A, which shows the tuneable speed of carbon devices from the slow to fast regime as the $s p^{2} \%$ increase in these structures.

The structural change for case $\mathrm{A}$ is also reflected in the LDOS accompanied by the peak position change and broadening. Fig. (5) shows the LDOS at the central cite for structures with different $s p^{2}$ bond concentrations. At a high concentration (Fig. 51 (a)) there are several high LDOS peaks inside $E_{\sigma}^{g}$. As the concentration decreases, LDOS peaks decrease being comparable in amplitude to LDOS corresponding to the $s p^{3}$ bands (Fig. 5 (b)). Finally, when the $s p^{2}$ bond concentration is less than $50 \%$ the LDOS peaks inside $E_{\sigma}^{g}$ become negligible compared to LDOS outside (Fig. [5 (c) and (d)). Also, the LDOS peaks move from the zero energy position with the conjugated phase decrease, effectively opening the gap. The broad features of LDOS suggest the possibility for hop-
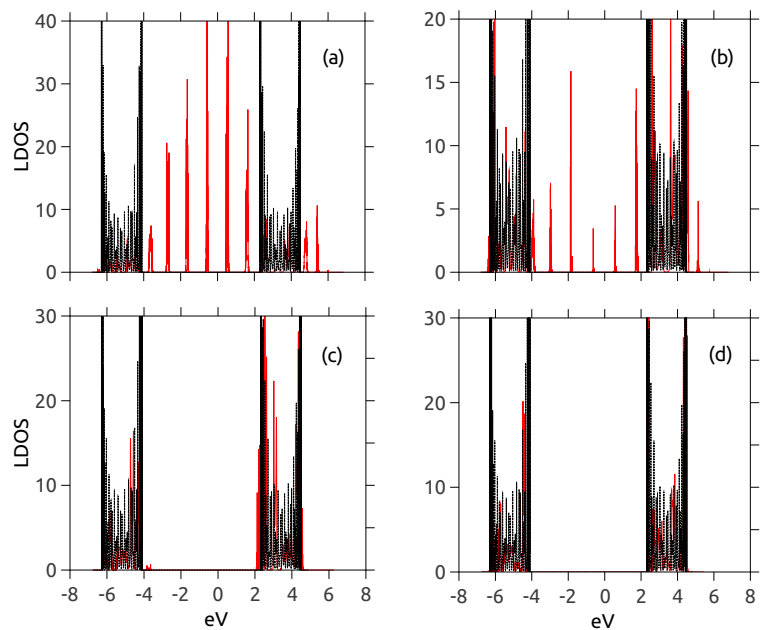

FIG. 5. LDOS (arbitrary units) for model A at the structure central cites for (a) $72 \%$, (b) $62 \%$,(c) $42 \% s p^{2}$, and (d) $24 \%$ $s p^{2}$ bonds (solid red curve) compared to that of pure $s p^{3}$ structure (dotted black curve).
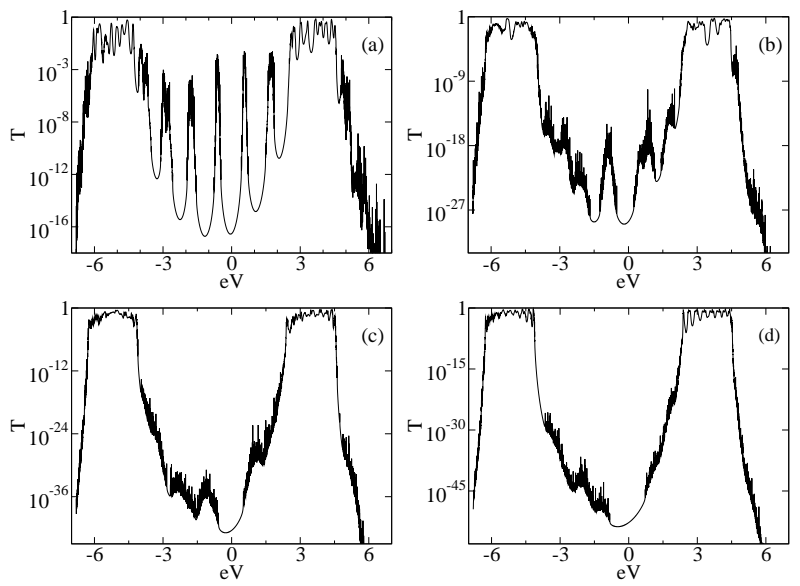

FIG. 6. $T(E)$ vs. $E$ for the (a) $72 \%$,(b) $42 \%$,(c) $24 \%$, and (d) $7.8 \%$ bond for the topological disorder considering model A. The energy axis is drawn relative to the Fermi energy.

ping transport in these structures, which is commonly observed in diamond-like carbon films [2, 21, 22]. On the other hand, the high LDOS filling the $s p^{3}$-gap region explains the metallic conduction in graphitic carbon films. Hence the observed conductance crossover in $a-C$ films with the increase of $s p^{2} C \%$ (or $N \%$ ) can be explained by the change of the characteristic time of the resonant states [25].

Besides the structural disorder we attempt to find the role of topological disorder on the transport properties of the structure. We know that topological disorder is more prominent in two-dimensional structures e.g. large $s p^{2}$ clusters however, the effects can also be simulated in quasi-one dimensional (small $s p^{2}$ clusters) structures. In the topologically disordered case for carbon superlattices or heterostructures, non-uniform $s p^{2}-C$ cluster sizes were distributed throughout the system in contrast to the non-topologically disordered case where all $s p^{2}$ clusters have the same size. Thus, it corresponds to so-called Anderson vertical disorder. The disorder parameter was determined using model A. As it can be seen from the Fig. 6] the topological disorder results in the decrease of the peak height and increase of the peak width and has a greater effect on the peaks located further from the Fermi level. The effect is more significant for structures with lower $s p^{2}$ concentration, so the relative change in the quasibound energy levels is high. In addition to that, new minor peaks appear in when the difference in energy levels induced by topological disorder is larger than the hopping structural disorder parameter. Topological disorder modifies the resonant tunneling conditions, changing the resonant energy conditions of consecutive wells resulting in a mismatch of the resonant levels. The amplitude of transmission peaks at resonant energies therefore decreases and the resonant peaks spread out with energy as resonance no longer occurs at a sharply defined energy. A resonance peak splits in subpeaks corresponding to the cluster number (4 in this work), where topological charac- 
teristic energy splitting $\delta \varepsilon_{t o p}$ is proportional to $\frac{\delta w}{w^{3}}$, where $w$ and $\delta w$ are the average cluster size and its characteristic variation. When $\delta \varepsilon_{t o p}$ is larger than the characteristic structural disorder variation determined via deformation potential as $\delta \varepsilon_{s t r}=\gamma \delta l$, where $\gamma$ is the deformation potential and $\delta l$ is the bond length variation, the subpeaks are distinguishable. With increasing structural disorder the subpeaks start to spread out and finally overlap when $\delta \varepsilon_{\text {top }}$ becomes smaller than $\delta \varepsilon_{s t r}$ with the elimination of the distinct subpeaks features due to the fact that the number of clusters is much smaller than the number of sites. The resonant peaks can therefore be tuned by disorder of both types which can be controlled to some extent experimentally through annealing or irradiation of the films [30]. Based on the bond length distribution included in the model, different ring structures e.g. five fold and seven fold symmetry, additional so-called topological disorder can be included subject to further study.

Conclusion: In this Letter, the importance of the nonlinear disorder vs. energy model (case A), an intermediate to uniform increase and decrease of disorder, is established. The theoretical analysis of this experimentally supported model discovers the possibility for a relative increase of the tunnel conductance in $s p^{3} C$-rich carbon due to the appearance of resonant transmission peaks close to the bands. Nitrogen doping, for example, could be used to vary the micro-structure of $a-C: N$ films as nitrogen incorporation increases the $s p^{2}-C$ ratio [18, 30]. The nitrogen concentration also influences the disorder [31]. Nitrogen doping is therefore not the same as conventional doping however it can still be exploited to modify the conductivity of $a-C$ films [18]. In this regard the result of the present study can be extended for doped carbon structures. A detailed study of nitrogen incorporation in $a-C$ structures has recently been carried out based on a model related to this work [32]. The lifetime of electrons can be nearly constant over a large range of $s p^{3} \mathrm{C} \%$ in carbon structures having a wide bandgap energy. Since the intensity and position of the resonant peaks can be tuned by the $s p^{2} C \%$ to $s p^{3} C \%$ ratio and associated disorder under this model, we can effectively predict the nano-electronic device properties in a wide range of undoped and doped carbon structures. This model can also explain the origin of observed resonant features in multilayered carbon systems [25].

The work was supported by the URC(WITs) and the NRF(SA) under the Nanotechnology Flagship Project. We would like to thank R. McIntosh for discussion.
[1] D. Beeman, J. Silverman, R. Lynds, and M. R. Anderson, Phys. Rev. B 30, 870 (1984).

[2] J. Robertson and E. P. O'Reilly, Phys. Rev. B 35, 2946 (1986).

[3] J. L. Bredas and G. B. Street, J. Phys. C, 18, 651 (1985).

[4] J. Tersoff, Phys. Rev. Lett. 61, 2879 (1988).

[5] G. Galli, R. M. Martin, R. Car, and M. Parrinello., Science 250, 1547 (1990).

[6] C. Godet, Diamond Relat. Mater. 12, 159 (2003).

[7] S. Kugler and I. Laszlo, Phys. Rev. B 39, 3882(1989).

[8] D. Dasgupta, F. Demichelis, C. F. Pirri, and A. Tagliaferro, Phys. Rev. B 43, 2131 (1991).

[9] D. R. McKenzie, D. Muller, and B. A. Pailthorpe, Phys. Rev. Lett. 67, 773 (1991).

[10] P. C. Kelires, Phys. Rev. Lett. 68, 1854 (1992).

[11] Th. Frauenheim, P. Blaudeck, U. Stephan, and G. Jungnickel, Phys. Rev. B 48, 4823 (1993).

[12] M. Tamor and W. Vassel, J. Appl. Phys. 76, 3823 (1994).

[13] P. Stumm, D. A. Drabold, and P. A. Fedders, J. Appl. Phys. 81, 1289 (1997).

[14] A. C. Ferrari and J. Robertson, Phys. Rev. B 61, 14095 (1999).

[15] G. Fanchini and A. Tagliaferro, Appl. Phys. Lett. 85, 730 (2004).

[16] G. Cherkashnin, O. Ambacher, T. Sciffer, and G. Schmidt, Appl. Phys. Lett. B 88, 172114 (2006).

[17] J. D. Carey and S. R. P. Silva, Phys. Rev. B 70, 235417 (2004).

[18] J. Robertson, and C. A. Davis, Diamond. Relat. Mater.
4, 441 (1995).

[19] F. Alibart, M. Lejeune, O. Durand Drouhin, K. Zellama, and M. Benlahsen, J. Appl. Phys. 108, 053504 (2010).

[20] K. Shimakawa and K. Miyake, Phys. Rev. Lett. 61, 994 (1988).

[21] A. Helmbold, P. Hammer, J. U. Thiele, K. Rohwer and D. Meissner, Philos. Mag., B, 72, 335 (1995).

[22] S. Bhattacharyya, Phys. Rev. B 77, 233407 (2008).

[23] P. Mikulski, J. Patyk, and F. Rozploch, J. Non-Cryst. Solids 176, 230 (1994).

[24] I. Martin and Ya. M. Blanter, Phys. Rev. B 79, 235132 (2009).

[25] S. Bhattacharyya, S. J. Henley, E. Mendoza, L. GomezRojas, J. Allam and S. R. P. Silva, Nature Materials 5, 19 (2006).

[26] A. Onipko, Phys. Rev. B 59, 9995 (1998).

[27] H. Efstathiadis, Z. Akkerman, and F. W. Smith, J. Appl. Phys. 79, 2954 (1996).

[28] U. Stephan, Th. Frauenheim, P. Blaudeck, and G. Jungnickel, Phys. Rev. B 50, 1489 (1994).

[29] M. Hjort and S. Stafström, Europhys. Lett. 46, 382 (1999).

[30] S. Bhattacharyya, M. Lubbe, P. R. Bressler, D. R. T. Zahn, and F. Richter, Diamond. Relat. Mater. 11, 8 (2000).

[31] G. Chimowa, D. Churochkin, and S. Bhattacharyya, Europhys. Lett. in press (2012).

[32] M. V. Katkov and S. Bhattacharyya, J. Appl. Phys. 111, 123711 (2012). 